\title{
16 cases of clinical treating experience after liver transplantation
}

\author{
Xue-Rong $\mathrm{He}^{1}$, Jin $\mathrm{Xia}^{2}$, Xiao-Mei Wang ${ }^{2}$, Jian-ping Gong ${ }^{2}$ and Ke You ${ }^{2 *}$ \\ ${ }^{1}$ Department of Surgery, The People's Hospital of Yongchuan District, Chongqing 402160, China \\ ${ }^{2}$ Chongqing Key Laboratory of Hepatobiliary Surgery and Department of Hepatobiliary Surgery, The Second Affiliated Hospital of Chongqing Medical University, \\ Chongqing 400010, China
}

\begin{abstract}
Objective: To discuss clinical nursing experiences after liver transplantation retrospectively. Methods From 2003 to 2013 in our hospital, a total of 16 patients with liver transplant, whose clinical nursing of patients experience would be summarized, and the important and difficult points in the process of it would be sum up.

Results: Out of 16 cases of liver transplantation one of them died soon due to intolerance for surgery, and two patients died postoperative due to respiratory system function failure. Other patients recovered well and discharged were from hospital. The percentage of living cases after one year of operation was up to $81.3 \%$.

Conclusion: The clinical nursing process after liver transplant is complex, and the technical operation is difficult, therefore it requires nurses in nursing processes to have comprehensive grasp and have skilled operation in order to improve the survival rate of patients and quality of life after liver transplantation.
\end{abstract}

\section{Introduction}

Liver transplantation that is considered one of the most complex procedures in modern surgery [1] is surgically implanted in a healthy liver into the patient's body, so that patients with liver disease will be well restored after a surgical treatment. It is used as a resource to guarantee the survival of patients with irreversible hepatic failure, when there is no other form of treatment available. As medical technology continues to evolve, the number of cases of liver transplantation is increasing. Although the clinical care and condition of the patient before transplant can impact the outcome after transplant [2], but clinical care after liver transplantation has become increasingly important. From April 2003 to May 2013 in our hospital, there were 16 cases of liver transplant, whose operations were successful implemented. And for liver transplantation clinical care we will make the following report.

\section{Clinical data}

There are 16 cases, 11 males and 5 females. The oldest is 71 years old, and the youngest is 33 years old. Mean age is 49 years. 5 cases of them were caused by cirrhosis. And 6 cases were attracted by primary liver cancer. Hepatitis caused 4 cases liver failure. The other one person suffered from polycystic liver disease, and he had made a combined kidney-liver transplantation. All sources of donor livers are DCD donations, with the approval of the hospital ethics committee to discuss all the cases. The longest hospital stays were 115 days, and the shortest were 16 days. Follow-up time: 1-10 years.

\section{Methods}

Collected clinical data and the nursing records in detail of the 16 liver transplant patients in our department from 2003 to 2013, and combined with relevant nursing experience of the nursing in clinical. For nursing key points of after liver transplantation are discussed and summarized. More effective and feasible way of nursing should be come up with, to increase the success rate of surgery and patients' survival rate.

\section{Results}

There are three patients died in hospital, including 1 case death from intolerance surgery a few days, and the other 2 cases died because of postoperative concurrent respiratory system failure. The others all ride out hospital stays and rehabilitation hospital discharge. One year after the operation, the living cases are up to $81.3 \%$.

\section{Discussion}

\section{Patients with psychological problems}

One patient had obvious psychological anxiety and panic. A 51 year old female patient had a postoperative unstable vital signs and she was transferred to ICU had a ICU syndrome after treatment (ICU syndrome is psychiatric symptoms when comprehensive treated for patients, caused by the treatment, the environment and many other factors. Antecedent symptom is insomnia, and symptoms are delirium and anxiety). Following psychological care has been taken for these symptoms: 1 closely observe the patient's mental state, to make an accurate assessment of active prevention. 2 a detailed description of the environment as well as ICU treatment conditions would be

Correspondence to: Ke You, Chongqing Key Laboratory of Hepatobiliary Surgery and Department of Hepatobiliary Surgery, Second Affiliated Hospital, Chongqing Medical University, Chongqing 400010, China, E-mail: youke_happy@126.com

Key words: liver transplantation, postoperative care

Received: December 28, 2016; Accepted: January 09, 2017; Published: January 12,2017 
explained to patients before entering ICU in order to allow the patient to eliminate strange and fear, so that patients have a positive state of mind, to establish confidence during the treatment period. 3 improve patient room facilities conditions, and let the patient have a comfortable environment for treatment, and the patient shall be properly arranged schedule time and treatment time. Wards should be kept quiet and clean environment, a certain vision and full of light. 4 to improve the operation of nursing skills, so nurses should familiar with the procedures and matters calmly deal with various unexpected situation. 5 actively encourage the patient's family and friends to involve in the communication with patient, explaining the patient doubts and anxiety problems, and encourage patients to actively accept treatment and enhance the ability of self-independence for those patient generating ICU syndrome.

\section{Treatment of postoperative complications}

Complications following transplantation can be caused by infections, recurrent disease such as primary biliary cirrhosis (PBC) or cancer and problems in other parts of the body that can take several years or decades to unfold [3]. The main complications include rejection after liver transplantation, all kinds of infection, bile duct complications, wound blood and liver transplantation to lose energy. This group includes 16 cases of postoperative complications mainly have different degree of fever, high blood pressure, wound infection, pain. There had three cases of patients with respiratory and circulatory system dysfunction, there were two cases died, including one case of renal dysfunction.

Control of refection and injection: Acute rejection may occur in up to $10 \%$ of liver transplant recipients [4]. It usually occurs 7 to 14 days after transplant $[5,6]$. Bacteria grow very well in ascetic fluid, and there is adiminished host immune response in chronic liver disease [7]. Thus, even in the absence of invasive abdominal procedures, many patients develop spontaneous bacterial peritonitis(SBP). The patients in the three cases of patients with high fever, varying degrees of refection, as we can use of antibiotics which had been effectively controlled. Serious infectious disease complications along the posttransplant course are associated with poor graft recover, prolonged stay in the ICU, and a high risk of multiorgan failure [7]. Acute rejection after liver transplantation is usually treated with large doses of immunosuppressants with severe toxic and side-effects, so it is imperative to find a safe and effective method for preventing and treating rejection [8].

Liver transplant patients had a single room isolated, and strictly limited access to staff, into the ward should make the appropriate protective isolation. Ward should be strictly sterilized items daily, indoor air disinfection with ultraviolet irradiation and daily monitoring of indoor air. Invasive procedures should ware sterile gloves, and made good respiratory humidification and atomization. Cough冈expectoration after tube drawing should be encouraged to prevent infection. Doing this drainage tube care, strict implementation of aseptic technique to prevent joint contamination, and can be used with antibiotics; doing basic care, attention to changes in body temperature and blood would be valued to prevent iatrogenic infection.

Stop bleeding: There are two cases in this group of patients in the drainage fluid was bloody, and after observing the situation improved, had no serious bleeding complications. The liver plays several key roles in blood coagulation being involved in both primary and secondary hemostasis. There are many vascular anastomosis after liver transplant can lead to bleeding, commonly in after 2 days. But abdominal bleeding is more common, which is early postoperative death reason. Patients should be closely monitored with blood pressure, central venous pressure, urine output. And observe the patient stool color, skin, without bleeding tendencies. If patients feel pain in the liver area, swelling, localized tenderness, rebound tenderness and other signs, accompanied by decreased blood pressure, pulse rate, etc, who should be promptly treated. Establishing vein channel, to facilitate blood rehydration, restore effective circulating blood volume. If you are diagnosed with active bleeding, in order to stop the bleeding should be decisive for abdominal operation.

Deal with biliary complications: Biliary complications are the most important source of complications after liver transplantation, and an important cause of morbidity and mortality [9], including bile duct fistula, bile duct stenosis and bile duct sludge formation. Bile decreased, color is green to liver dysfunction performance. To keep the T-tube drainage unobstructed, T-tube must be protected properly. Once reduced bile drainage is reduced, wound with yellow exudates should be dealt immediately. One case reported a small amount of yellow liquid oozing out of patients wound. 2 patients drainage volume decreased after active treatment and are back to normal.

\section{Summary}

Liver transplantation has become an effective and valuable option for a patient with end-stage liver disease and hepatocellular Carcinoma [10]. It has opened an exciting opportunity to study liver regeneration in humans [11]. But liver transplant patients with severe illness, surgery complicated condition changes quickly. Through this group of patients with 16 cases of liver transplantation in patients with clinical observation and care, we realized that: (1) for postoperative patients, psychological nursing is important to ensure rapid recovery; (2) the control of infection and rejection is the key to the patients with postoperative rehabilitation; (3) to deal with the postoperative complication and can improve the patients survival rate and reduce pain. After liver transplantation, clinical care and observation are important components to surgery.

\section{Funding}

The project is supported by the National Natural Science Foundation of China (No: 81370580 and 81200329).

\section{References}

1. Mendes KDS, Galvao CM (2008) Liver transplantation: evidence for nursing care. Rev Lat Am Enfermagem 65: 456-23. [Crossref]

2. Grogan TA (2011) Liver transplantation: issues and nursing care requirements. Crit Care Nurs Clin North Am 23: 443-456.[Crossref]

3. Cholongitas E, O’Beirne J, Betrossian A, Senzolo M, Shaw S, et al. (2008) Prognostic impact of lactate in acute liver failure. Liver Transpl 14: 121-122.[Crossref]

4. Levy G, Graxi GL, Sanjuan F, Wu Y, Mühlbacher F, et al. (2011) 12-month followup analysis of a multicenter, randomized, prospective trial in denovo liver transplant recipients (LIS2T) comparing cyciosporinemicroemulsion (C2monitoring) and tacrolimus. Liver transpl 12: 1464-1472. [Crossref]

5. Rudow DL, Goldstein MJ (2008) Critical care management of the liver transplant recipient. Crit Care Nurs Q 31: 232-243.[Crossref]

6. Macnaughtan J, Jalan R (2015) Clinical and pathophysiological consequences of alterations in the microbiome in cirrhosis. Am J Gastroenterol 110: 1399-1410. [Crossref]

7. Feltracco P, Barbieri S, Galligioni H, Michieletto E, Carollo C, et al. (2011) Intensive care management of liver transplanted patients. World J Hepatol 3: 61-71.[Crossref]

8. Chi-Dan Wan, Rui Cheng, Hong-Bo Wang et al. (2010) Immunomodulatory effects of mesenchymal stem cells derived from adipose tissues in a rat orthotopic liver transplantation model. Hepatobiliary Pancreat Dis Int 2:75-53.[Crossref] 
9. DuailibiDF, Ribeiro MAF (2010) Biliary Complications Following Deceased and Living Donor Liver Transplantation: A Review. Liver Transpl 25: 450-231. [Crossref]

10. Coscia C, Saxton E, Dickinson S (2016) Postoperative Care of a Liver Transplant
Recipient Using a Classification System: Type A (Stable) Versus Type B (Unstable) Crit Care Nurs Q39: 252-266. [Crossref]

11. Taki-Eldin A, Zhou L, Xie HY, Zheng SS (2012) Liver regeneration after liver transplantation. EurSurg Res 48: 139-153. [Crossref]

Copyright: (C2016 He XR. This is an open-access article distributed under the terms of the Creative Commons Attribution License, which permits unrestricted use, distribution, and reproduction in any medium, provided the original author and source are credited. 